\title{
Recognition of Apoptotic Cells by Viruses and Cytolytic Lymphocytes: Target Selection in the Fog of War
}

\author{
David Schwartz and Sujatha lyengar
}

\begin{abstract}
Viruses and cytolytic lymphocytes operate in an environment filled with dying and dead cells, and cell fragments. For viruses, irreversible fusion with doomed cells is suicide. For cytotoxic T lymphocyte and natural killer cells, time and limited lytic resources spent on apoptotic targets is wasteful and may result in death of the host. We make the case that the target membrane cytoskeleton is the best source of information regarding the suitability of potential targets for engagement for both viruses and lytic effector cells, and we present experimental evidence for detection of apoptotic cells by HIV, without loss of infectivity.
\end{abstract}

Keywords: apoptotic, cytoskeleton, lytic synapse, HIV, CTL, NK

"I share Alfred Nobel's conviction that war is the greatest of all human disasters. Infectious disease runs a good second."

-Peter C. Doherty

\section{Target Selection in the "fog of war"}

C ARL VON ClAUSEWITZ is credited with first articulating the concept of nebel des krieges ("fog of war") in his 1873 treatise, On War, to describe the uncertainties surrounding enemy status in the midst of war, and the difficulties in identifying appropriate targets. Applying the battlefield metaphor to viral infection and the anti-viral immune response, we can appreciate the difficulties faced by both viruses and anti-viral lytic effector cells in discriminating between suitable and unsuitable targets. In addition to healthy, uninfected, virus susceptible cells, and viable infected cells (appropriate targets for, respectively, infectious virus and cytotoxic lymphocytes), the killing field contains apoptotic uninfected and infected cells, and cell blebs. Uninfected cells may be triggered to apoptosis through antigen-specific or nonspecific exhaustive proliferation, or non-cognate contact with infected cells, and by released cytokines, or antiviral chemotherapy $(13,30,47,51,57,60,64,87)$. Infected cells may also undergo intracellular viral induced apoptosis, or programmed death after lethal encounters with natural killer $(\mathrm{NK})$ or cognate cytotoxic $\mathrm{T}$ lymphocytes (CTLs). Apoptotic membrane blebs can contain surface viral receptors and/or Class I MHC molecules presenting viral peptide antigens $(31,55,73,82,89)$.

\section{Viral Recognition and Avoidance of Fusion with Apoptotic Targets}

Viruses-especially retroviruses-would benefit from the ability to avoid infecting or irreversibly fusing with dying cells by detecting apoptotic target membrane features before irreversible fusion. This would prevent dead-end entry into doomed hosts, which are incapable of supporting productive infection. For example, HIV and SIV replicate primarily in a setting of increased uninfected $\mathrm{CD}^{+}$lymphocyte proliferation and turnover, marked by activation-induced cell death due to Fas and other contact-triggered suicide initiators, including uninfected cell contact with infected cells $(13,30,51,57,60,64,87)$. Most leukocytes die within $12 \mathrm{~h}$ of initiating programmed cell death in vitro, and cell machinery required for viral integration, replication, assembly, and

Jurist Research Department, Hackensack University Medical Center, Hackensack, New Jersey.

(c) David Schwartz and Sujatha Iyengar 2020; Published by Mary Ann Liebert, Inc. This Open Access article is distributed under the terms of the Creative Commons Attribution Noncommercial License (http://creativecommons.org/licenses/by-nc/4.0/) which permits any noncommercial use, distribution, and reproduction in any medium, provided the original author(s) and the source are cited. 
budding is compromised much sooner (23). This poses an existential problem for retrovirus with a minimum single replication cycle of $\sim 30 \mathrm{~h}$ under optimal conditions (61). Moreover, in vivo, apoptotic cells can be eliminated rapidly, due to surveillance and removal by phagocytes recognizing "eat me" signals such as everted phosphatidyl serine (PS). Exosomes and apoptotic blebs may contain surface MHC, CD4, virus binding chemokine receptors (CKRs), and damageassociated molecular pattern proteins.

Since cells undergoing programmed death less than $24 \mathrm{~h}$ after irreversible virus-cell fusion are "sterile," preservation of infectivity after encountering apoptotic cells would confer a selective advantage for virions in an HIV infectious swarm. Consider two HIV virions, one of which is capable of avoiding fusion with apoptotic membranes, with no other differences in viral fitness. Assuming stochastic encounters, replication in a cell population containing $10 \%$ apoptotic targets would result in a swarm comprising overwhelmingly (>99.999\%) apoptosis discriminating virions after 35-40 cycles. Most cases of HIV infection are only confirmed after this acute phase, so we would expect essentially all viruses recovered from patients to exhibit this capacity, even assuming infection with a predominantly non-discriminating swarm, and discounting the high mutation rate of HIV, which rapidly generates new geno-phenotypes under selective pressure.

How might this discrimination occur? Although externalized PS is well established as an apoptotic feature marking cells for phagocytosis, it is also transiently exposed on activated cells that are the best hosts for HIV and herpes viruses. Investigators recently showed that initial HIV envreceptor engagement induces $\mathrm{Ca}++$ flux-triggered PS externalization, which, in turn, dramatically enhances viral fusion and entry $(16,95)$. In addition, many tumors upregulate surface PS (20), yet they remain susceptible-even preferentially so - to a variety of oncolytic viruses (66). PSmediated "apoptotic mimicry" refers to enveloped pathogens carrying everted PS exploiting cellular receptors for uptake of apoptotic debris, and indirect induction of interleukin (IL)-10 and other immunosuppressive cytokines by phagocytes after ingestion of infected cells or cancer cells exhibiting externalized PS (9). These are not examples of surface PS signaling "stay away!" to intracellular pathogens. These observations make it highly unlikely that exposed PS is the signal preventing irreversible viral fusion with apoptotic cells. By contrast, defects in cytoskeletal and associated protein function represent an ideal barrier to cellfree and cell-associated viral fusion.

We published the first demonstration of HIV (or any virus) requiring target cell actin-myosin cytoskeletal function for fusion-mediated entry, specifically, co-capping of CD4 and CKRs (36). Our results with cell-free virions were subsequently confirmed for cell-associated HIV $(40,41)$, and for several other types of virus $(59,85)$. Given the paucity of HIV envelope fusion spikes ( $<100$ per virion), and the need for multiple trimeric spikes to simultaneously engage their receptors and insert envelope fusion components to efficiently mediate membrane penetration, we have argued that the use of a single cell surface receptor for virus places a much lower stochastic burden on binding than the requirement for simultaneous dual receptor binding. Single receptor binding should, therefore, facilitate spread (38). However, the great majority of HIV and SIV strains recovered both in vivo and in vitro require dual binding of co-capped CD4 plus CKR to trigger virus-cell membrane fusion. Since HIV appears to have evolved a more stringent requirement for actin cytoskeleton-mediated dual receptor co-capping as compared with ancestral SIV (52), we reasoned that this must confer a selective advantage over use of only a CKR-namely, avoidance of inappropriate $\mathrm{CD}^{-}$and/or quiescent or dying $\mathrm{CD}^{+}$targets that are incapable of supporting viral replication.

Using SIV, we demonstrated this selective advantage for actin-dependent receptor co-capping by the emergence of predominant co-receptor dependence several generations after infection in vitro with a single receptor-dependent strain (37). Similar emergence of predominantly dual receptor SIV after infection with a single CKR-dependent virus had previously been observed in macaques $(21,46,74)$, but those in vivo studies were complicated by issues of potential selective pressure from immune responses and depletion of target cell populations. Our studies revealed rapid in vitro evolution toward dual receptor usage, independent of host defense mechanisms or exhaustion of host target supply. We hypothesized that selection of $\mathrm{CD}^{+}$hosts was a major evolutionary driver of initial CD4 binding, proposing greater intrinsic replication competence of $\mathrm{CD} 4^{+}$ versus $\mathrm{CD}^{-} \mathrm{T}$ cells. In addition, if receptor co-capping is a marker of non-apoptotic cells, this could explain the retention of the CKR requirement (as opposed to CD4 only). Further studies of HIV-cell membrane fusion have implicated endocytosis and target cell filopodia surface transport $(15,50)$. These mechanisms, like receptor co-capping, require a functional target cell cytoskeleton. Moreover, exploitation of the host cell cytoskeleton for entry is a feature of many other types of virus $(68,80,86)$.

Since defective host cell membrane cytoskeletal function would be a better marker of apoptosis than exofacial PS, with respect to retrovirus encountering apoptotic host cells, we hypothesized that cytoskeletal defects in co-receptor capping would prevent irreversible fusion. Surprisingly, although cytoskeletal functions play an essential role in the morphological changes seen during apoptosis (22), little has been published as to when surface receptor capping is lost during lymphocyte programmed cell death. We, therefore, generated data showing that within $2-3 \mathrm{~h}$ of Fas engagement, apoptotic cells fail to cap CXCR4 when exposed to CD4/CXCR4 binding HIV envelope gp120 (below, Fig. 1) or intact HIV (not shown). Non-adherent day 3 Phytohaemagglutinin (PHA)-activated peripheral blood mononuclear cells (PBMCs) were exposed to $100 \mathrm{ng} / \mathrm{mL}$ Fas cross-linking anti-Fas IgM mAb for $3 \mathrm{~h}$, then placed in droplets onto Poly-L-Lysine covered replicate glass slides, and exposed to $5 \mu \mathrm{g} / \mathrm{mL}$ CHO cell produced recombinant CXCR4-tropic rgp120 (HIV IIIB strain, ImmunoDiagnostics, Inc., MA). At 15, 30, 60, and 90 min after addition of rgp120, cells were fixed with paraformaldehyde; then, they were stained with FITC-conjugated secondary Ab anti-CXCR4 (green) and PE-conjugated Annexin V (red). Confocal overlapping images were originally obtained at $40 \times$ and $100 \times$ magnification.

At $15 \mathrm{~min}$ (Fig. 1A), none of the lymphocytes showed evidence of CXCR4 surface capping, and roughly half the cells exhibited diffuse red labeled Annexin $\mathrm{V}$ binding to everted PS, indicative of apoptosis. By $30 \mathrm{~min}$ (Fig. 1B), a significant proportion of the non-apoptotic (green only) cells 

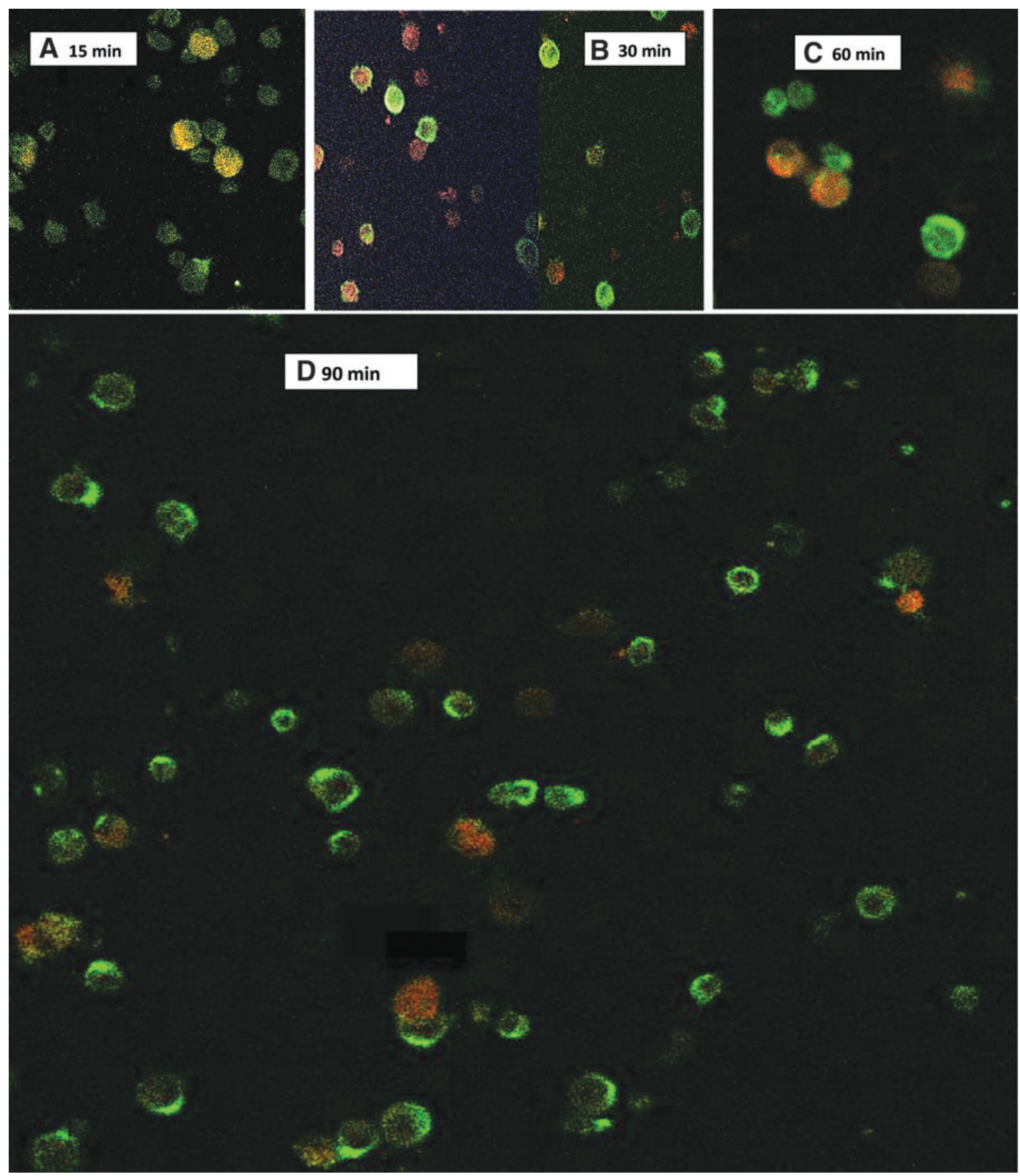

FIG. 1. Apoptotic cells (red stained for PS) fail to co-cap green stained CXCR4 at 15 (A), 30 (B), 60 (C), or 90 (D) minutes, whereas non-apoptotic (green only) cells show progressively increased and localized capping over the same 90 minute time period.

exhibited surface CXCR4 clustering or partial capping, visible as a bright circumferential green rim or crescent. Apoptotic cells staining red for Annexin V showed only diffuse green stippling. At $60 \mathrm{~min}$ (Fig. 1C), many green non-apoptotic cells showed clear evidence of CXCR4 polar capping. By contrast, none of the red apoptotic cells exhibited polarized or even partially polarized CXCR4. Finally, after $90 \mathrm{~min}$ (Fig. 1D), most of the non-apoptotic green cells had formed CXCR4 caps or pseudopods, whereas dual staining Annexin V positive cells appeared predominantly red, retaining the diffuse distribution of green CXCR4 seen at the $15 \mathrm{~min}$ time point.

\section{Retained Infectivity of HIV Bound, but Not Fused, to Apoptotic Cells}

Retention of infectivity for viruses that bind apoptotic cells without entering is crucial to our hypothesis. Loss of infectivity after non-fusion encounters would not confer a selective advantage over non-discriminating virus strains. We, therefore, tested this in transmission experiments. Whole PBMCs were stimulated for 3 days with PHA and IL-2 to generate blast forms suitable for infection. Untreated control cells were maintained without further manipulation before use in infection-transmission assays. For $36 \mathrm{~h}$ before 
HIV exposure, the remaining cells were continuously cultured in $25 \mu \mathrm{M}$ AZT $+25 \mu \mathrm{M} 3 \mathrm{TC}$ to block intracellular HIV reverse transcription. Drug-treated or -untreated cells were then split into two groups. One was incubated with anti-Fas $\operatorname{IgM}(2 \mu \mathrm{g} / \mathrm{mL})$ for $3 \mathrm{~h}$ to induce apoptosis. The other group was not. The anti-Fas-triggered population was labeled for dead cells (Miltenyi Dead Cell kit), which were removed by immunomagnetic column filtration. The recovered live cells were then positively immunoselected for expression of PS bound to Annexin V. Meanwhile, the group of PHAstimulated cells not exposed to anti-Fas was labeled with Annexin $\mathrm{V}$ and dead cell reactive mAbs, and it was negatively column selected to recover the pass-through nonapoptotic viable population.

Identical but separate pools of PHA-activated, AZT +3TC-treated cells were used to obtain non-apoptotic versus anti-FAS-triggered apoptotic cells. In this way, we addressed the theoretical possibility that cells most susceptible to HIV infection after PHA stimulation are also the most susceptible to Fas-induced apoptosis. By not simply separating one pool of anti-Fas-treated cells into apoptotic versus non-apoptotic populations, we avoided the potential pitfall of obtaining a non-apoptotic activated population in which the most infectable cells had already been driven into apoptosis by prior Fas cross-linking.

Populations of PHA-activated PBMCs, highly enriched for either non-apoptotic cells or viable apoptotic cells, were exposed to $\sim 200 \mathrm{ng}$ p24 equivalents of CCR5 tropic HIV-1 $\mathrm{BaL}$ at $5^{\circ} \mathrm{C}$ (binding permissive) or $37^{\circ} \mathrm{C}$ (binding and fusion permissive). After $1 \mathrm{~h}$ of viral exposure, cells were washed $\times 3$, and $1 \times 10^{5}$ cells were plated into quadruplicate $1 \mathrm{~mL}$ "rescue" cultures containing $5 \times 10^{5}$ PHA-stimulated, non-apoptotic, autologous PBMCs that had not received antiretroviral treatment. These rescue cultures were sampled, in parallel, for supernatant p24 at days 1, 3, 5, 7, and 10 to assess viral transmission from the various HIV-exposed cell populations, and expansion in untreated autologous PHAactivated PBMCs (Fig. 2).

As shown, non-apoptotic control cells, not pre-treated with antiretroviral drugs, and exposed to HIV at cold or warm temperatures, were able to transmit HIV to fresh PBMC cultures. However, when non-apoptotic cells were pre-treated with AZT $+3 \mathrm{TC}$, they failed to transmit detect- able infection to the surrounding susceptible amplifying culture cells, indicating complete internalization and subsequent inactivation of all surface bound virus remaining after the third wash. Presumably, HIV bound at $5^{\circ} \mathrm{C}$ was so intimately bound to the cell membrane that fusion was completed on deposition into the $37^{\circ} \mathrm{C}$ rescue cultures before any fresh PBMCs were encountered. By contrast, after three washes, all groups of apoptotic cells, with (shown) or without (not shown) antiretroviral pre-treatment, remained associated with enough infectious and transmissible HIV to speed robust viral growth in rescue cultures. This demonstrates lack of internalization by apoptotic cells, and retention of bound infectious virions at the apoptotic cell membrane surface. Though not critical for our hypothesis, CD4-specific HIV binding was confirmed by pre-treatment with CD4 blocking mAb Leu3a before HIV exposure. All groups pre-treated with Leu3a, including apoptotic cells at $5^{\circ} \mathrm{C}$ and $37^{\circ} \mathrm{C}$, failed to transmit detectable infection to rescue cultures, demonstrating prevention of virus binding.

\section{Cytotoxic Lymphocyte Recognition and Avoidance of Apoptotic Targets}

The ability to quickly detect and avoid engagement with apoptotic targets would also be highly advantageous for cytotoxic effector cells, engaged in a race with exponentially replicating virus $(62,90,91)$. Lymphocyte-mediated cytolysis entails receptor detection of surface target cognate antigen, a time-consuming process of membrane spreading and probing for rare (as few as 1-5) surface cognate antigens. Recognition is followed by effector cell polarization, formation of a cytolytic synapse, and assembly of a perforinbased pore through which pre-formed granzymes are injected. In addition, surface membrane Fas on target cells may be engaged and cross-linked by CTL surface FasL, triggering granzyme-independent apoptotic pathways. Each of these steps takes precious time and metabolic energy on the part of CTL or NK cells, which typically spend several minutes in membrane contact with targets. The actual completion of target cell apoptosis and/or lysis can take much longer, and cytolytic effectors are believed to disengage in many cases before the fate of a $\mathrm{CD}^{+} \mathrm{CTL}$ or NK-targeted tumor or virus-infected cell is sealed $(14,33,39)$, although

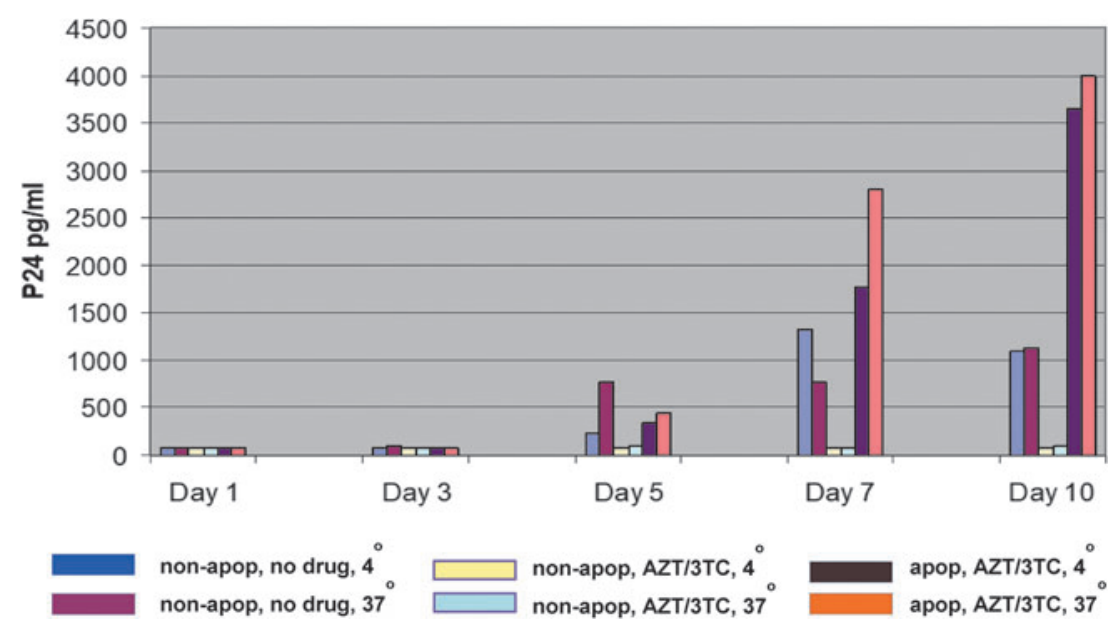

FIG. 2. HIV bound to apoptotic cells is not internalized and remains infectious. 
some in vivo experiments yielded synaptic dwell times averaging $6 \mathrm{~h}$ for immunogenic tumors and $21 \mathrm{~h}$ for cognate killing of non-tumor targets (8). These different rates of killing and cytotoxic synapse duration reflect NK and CTL heterogeneity and density, target heterogeneity and density, mobility within tissue fields, and volume of killing fields $(28,29,42)$.

Importantly, CTL and NK cells carry out their "seek and destroy" missions in a complex mixed environment of healthy, diseased, dying, and dead cells. Potential targets may be undergoing apoptosis due to virus-triggered programmed death, anti-viral drug therapy, or after prior lethal hits from effector CTLs and NKs. In the case of tumor cells, radiation therapy and vascular insufficiency are additional triggers of apoptosis. How can effector cells avoid wasteful engagement with dying targets? More information is available for $\mathrm{NK}$ cells than for $\mathrm{CD}^{+} \mathrm{CTL}$. Direct interaction with $\mathrm{MHC}$ class I leads to inhibitory phosphorylation of NK cell KIRs $(34,54)$. Disengagement of NK cells carrying out antibody-dependent cell-mediated cytotoxicity (ADCC) occurs via shedding of NK surface FcR (CD16) after repeated engagement $(24,77)$. However, neither of these avoidance and release mechanisms explains NK disengagement from non-ADCC targets with downregulated class I. Likewise, the mechanisms responsible for $\mathrm{CD} 8^{+} \mathrm{CTL}$ avoidance of, abortive engagement with, or detachment from targets are poorly defined.

Broadly speaking, there are two conceptually distinct strategies for effector-target detachment or lack of initiation of synaptic engagement. One relies on immunologic synapse (IS) disassembly due to summed receptor signals, or an internally timed cascade, leading to shedding or recycling of effector cell receptors. Such mechanisms entail programs, intrinsic to the effector cell, that are irreversible at some point post-engagement, regardless of target status. The second general strategy posits continuous sensing of target cell membrane properties to detect the presence, or absence, of some component that is necessary for synaptic initiation, maintenance, or detachment. In this model, the health status of the target, rather than (or, in addition to) a pre-determined effector cell program, signals when it is time for CTLs or NKs to release and move on.

Experimental data exist for both effector-intrinsic and target-intrinsic models. In vivo and lymph node in situ studies suggest that CTLs move rapidly from cell to cell, delivering short "hits" that are insufficient, individually, to trigger cell death, with multiple closely spaced CTL encounters required to induce death in virus-infected (33) or tumor $(14,39)$ cells. The evolutionary value of such effectormediated early release can be inferred from studies showing that prolonged NK lytic IS engagement results in excessive NK inflammatory cytokine secretion, creating a potentially lethal "cytokine storm" $(18,39,49)$. However, the time between the last hit and actual cell death could also reflect target mediated changes that deter any further surface interaction with effectors. In fact, when NK targets were pretreated with caspase inhibitors, detachment was delayed more than fivefold (from 8 to $>40 \mathrm{~min}$ ), with consequent increased NK secretion of IL-2, tumor necrosis factor, interferon-gamma, and inflammatory chemokines (39).

The need to avoid catastrophic cytokine storm may explain premature release from still viable targets via mechanisms that are intrinsic to lytic effectors. Target cell caspase-dependent release mechanisms simultaneously prevent dangerously prolonged IS dwell times, as well as inefficiently redundant effector "hits." How, then, is intracellular caspase release detected at the target cell's outer membrane where effectors engage? Everted PS is one consequence of caspase activation, but we have already noted, with respect to viruses, the inadequacies of exofacial PS as a suitable marker of irreversibly doomed targets. It is also less than ideal for marking CTL targets as irreversibly damaged, since it is upregulated on some infected cells that are appropriate targets, and on many viable tumor cells $(9,16,20,66,67,95)$. Phagocytosis of infected or malignant cells expressing surface PS does induce local immune tolerance, and is an example of co-opted homeostatic effector down-modulation, but its indirect, cytokine-mediated mechanism of M2 induction makes it unsuitable as a contactdependent signal of target cell apoptotic status (9). By contrast, for cytotoxic lymphocytes as for viruses, the target cell membrane is the ideal transducer of information regarding potential target cell health status.

Recently, Kumari et al. (45) demonstrated that the duration of non-lytic stimulatory IS between $\mathrm{CD}^{+} \mathrm{T}$ helpers and antigen presenting cells (APCs) is controlled by maintenance of $\mathrm{CD}^{+} \mathrm{T}$ cell cytoskeleton-dependent tension in the plane of the activation synapse. This decays as $\mathrm{CD}^{+} \mathrm{T}$ cell Wiskott-Aldrich syndrome protein (WASP) is degraded, consistent with previous findings on the role of WASP in APC-T cell IS stability (56). Effector cell membrane tension may be monitored by the co-localized mechanosensory protein CasL, which is phosphorylated on conformational stretching $(75,94)$. This represents effector cell programmed release of the CD4 T cell stimulatory synapse. Several groups have shown that $\mathrm{CD}^{+} \mathrm{CTLs}$ also exert lateral force in the plane of the target cell membrane, and that this tension is required for the efficient formation and function of lytic synapses, including perpendicular indentation of the target membrane by CTL microprojections and perforin pore formation $(7,48,79)$. In fact, the T cell receptor (TCR)CD3 complex has been described as a mechanosensor $(17,26,35,93)$, operating as an immunotope-discriminating "catch bond," exquisitely sensitive to physiologic shear forces $(25,76,92)$.

However, synapse tension has been elucidated with respect to the cytolytic effector's tension-generating cytoskeletal machinery rather than the target cell's, Newton's Third Law dictates that effector cell tension requires equal opposing resistance (i.e., tension) from the target membrane. Indeed, required cytoskeletal reorganization in APCs and tumor cells at the stimulatory and lytic IS, respectively, has been demonstrated $(4,5,17,35,56,93)$. Moreover, confirming and expanding much earlier reports by Roozemond et al. $(69,70-72)$ that excessive fluidity or rigidity of target membranes interferes with NK killing, the susceptibility of cancer cells to NK and CTL lysis has recently been found to vary with membrane stiffness and the presence, activation state, and functional distribution of tumor cytoskeletal and intercellular adhesion molecules $(1,2,53,81)$. Results are not entirely consistent across systems with respect to the impact of changes in membrane stiffness, but supporting matrices differ dramatically in their inherent rigidity and the resulting visco-elasticity of supported cells $(63,65)$. It is also worth 
noting that mitotic cells, in general, and mitotic tumor cells, in particular, exhibit spherical rounding and increased membrane stiffness $(12,65,83)$. This may prevent formation of lytic synapses-which typically require $20-100 \mu \mathrm{m}^{2}$ of flat surface area interface-thereby contributing to escape from immune surveillance and/or killing.

Involvement of target cytoskeletal status in regulation of immune synapses with APCs and cancer cells strongly suggests a role for some of these same molecules in apoptotic target recognition. The full array of cytoskeletal components and adhesion molecules has been implicated in very early, even initiating, apoptotic membrane changes, as well as subsequent stages of delamination, blebbing, and organelle disintegration $(22,27,84)$. For example, during Drosophila development, in some cells the earliest initiating phase of apoptosis entails actin-mediated movement of the nucleus to the cell periphery (6), preceding caspase activation by damaged mitochondria. Clearly, such polarization might be detectable at the cell surface. However, mechanisms of programmed death operating during development may not be fully applicable to cytolytic processes. Most studies report loss of mitochondrial transmembrane potential and/or caspase cascade as initial CTL target cell responses, leading to early cleavage of actin and other cytoskeletal proteins. Moreover, granzyme B, injected through CTL perforin pores, was reported to directly cleave $\alpha$-tubulin, filamin, and gelsolin components of the cell cytoskeleton $(3,10,22,32,51,58,82,89)$; whereas Fas-mediated apoptosis induces early caspase 3 dependent cleavage of gelsolin, leading to actin filament disintegration (44). Conversely, cleaved $14 \mathrm{kD}$ fragments of actin, and even cytoskeleton mechanical distortion, accelerate and potentiate apoptosis (22). Thus, early disruption of the cytoskeletal architecture serves as a positive feedback loop driving irreversible death programs.

Two early features of apoptotic cells mediated by cytoskeletal changes are rounding and roughening (ruffling) of the cell membrane (43). Roughening is also a feature of malignancy (88), and it may interfere with cytolytic synapse architecture and pore formation in CTL-resistant tumor cells. Rounding, as noted earlier, if sufficiently severe and rigid (as in the case of apoptotic blebs), could thwart the formation of lytic synapses. Apoptosis is also reported to increase membrane stiffness, although this is not a universal finding, and may vary with cell type, method of measurement, and stage of death. As for tumor cells, increased stiffness could impede the ability of CTLs and NK cells to form a planar synaptic interface if it prevented adequate flattening deformation of curved membranes. Again, studies positively correlating activation of cytolytic mechanisms with target membrane stiffness have employed artificial, rigid supports, so issues of membrane curvature and lytic synapse geometry have not really been addressed.

Overall, the most likely mechanisms for apoptotic membrane prevention of effective lytic synapse formation are (1) a lack of appropriately organized, activated, or recruitable target cytoskeletal components and associated ligands to establish and/or stably anchor the maturing lytic synapse; (2) inability to generate adequate complementary opposing lateral forces in the target membrane as required for transmission of mechano-transduced signals to the effector cell via cognate catch bond receptors.

\section{Summary and Conclusions}

We have outlined various related obstacles facing viruses and cytolytic lymphocytes seeking suitable targets enshrouded in the "fog of war." We describe significant, even existential, challenges posed by the presence of apoptotic targets and cell fragments, and we hypothesize that detection of early apoptotic changes in cytoskeletal function an$\mathrm{d}$ /or composition constitutes ideal markers for external determination of potential target cell status. We present experimental evidence that HIV virions bind specifically to CD4 on apoptotic cells, but, in the absence of triggered coreceptor capping, fail to fuse with these doomed hosts. We further show that these CD4-bound virions remain infectious at the apoptotic cell surface, and productively infect fresh, non-apoptotic targets, thereby giving them a potent survival advantage.

Likewise, early and recent studies on the importance of the target cell cytoskeleton for functional lytic synapse formation are consistent with the idea that cytoskeletal alterations during early programmed cell death could deter initial or prolonged engagement of CTLs and NK effectors. We note that increased fixed curvature, stiffness, and roughness of the early apoptotic and mitotic cancer cell membranes, and cell blebs and fragments, may interfere with formation of a planar lytic synapse. Alternatively, defects in target cytoskeletal function, or excessive membrane fluidity may prevent appropriate generation of lateral tension at the IS.

Elucidating in detail the role of apoptotic target membrane components in restricting lytic contact and dwell time could provide new avenues for therapeutic intervention, and it could improve our understanding of the kinetics of cellmediated virus and tumor clearance. For example, a recent study of engineered chimeric antigen receptor (CAR) T cells revealed a less symmetric, more fragmented, and less wellorganized lytic IS, with minimal LFA-1 dependence, compared with conventional CD8 TCR synapses (19). Key findings were faster, more efficient pore formation and granzyme injection, and quicker detachment by CAR T cells. Understanding target membrane contributions to the observed kinetic differences between CAR and TCR T cell target engagement may inform the rational design of future generation CAR T cells, allowing them to ignore apoptotic targets, while exhibiting increased ability to focus firepower on elusive stem cancer cells.

More broadly, it is clear that cancer cells and intracellular pathogens have evolved evasive strategies that co-opt mechanisms of immune homeostasis, such as the induction of immune checkpoint inhibitors, leading to chronic persistence and/or T cell exhaustion. It would not be surprising, therefore, whether some engagement-deterring features of apoptosis were, likewise, co-opted by tumors and virusinfected cells. Indeed, externalized PS has already been adduced as a generalized example of "apoptotic mimicry" exploited by tumors and many intracellular pathogens (9). Viruses remodel the host cytoskeleton to impair immune recognition (78), and inflammation causes increased rigidity in primary APCs (11). Future studies should explore effector-apoptotic target interactions in in vivo or in ex vivo systems that replicate stroma with respect to crucial elements of stiffness, three-dimensional contact, oscillatory shear stress, cytokine milieu, and physiologic hypoxia. 


\section{Author Disclosure Statement}

No competing financial interests exist.

\section{Funding Information}

NIH 5R21 AI084683-02 awarded to S.I.

\section{References}

1. Abouzahr S, Bismuth G, Gaudin C, et al. Identification of target actin content and polymerization status as a mechanism of tumor resistance after cytolytic $\mathrm{T}$ lymphocyte pressure. Proc Natl Acad Sci U S A 2006;103:1428-1433.

2. Abouzahr-Rifai S, Hasmim M, Boukerche $\mathrm{H}$, et al. Resistance of tumor cells to cytolytic $\mathrm{T}$ lymphocytes involves Rho-GTPases and focal adhesion kinase activation. J Biol Chem 2008;283:31665-31672.

3. Adrain C, Duriez PJ, Brumatti G, Delivani P, Martin SJ. The cytotoxic lymphocyte protease, granzyme B, targets the cytoskeleton and perturbs microtubule polymerization dynamics. J Biol Chem 2006;281:8118-8125.

4. Al Absi A, Wurzer H, Guerin C, et al. Actin cytoskeleton remodeling drives breast cancer cell escape from natural killer-mediated cytotoxicity. Cancer Res 2018;78:56315643.

5. Al-Alwan MM, Rowden G, Lee TD, West KA. The dendritic cell cytoskeleton is critical for the formation of the immunological synapse. J Immunol 2001;166:1452-1456.

6. Ambrosini A, Rayer M, Monier B, Suzanne M. Mechanical function of the nucleus in force generation during epithelial morphogenesis. Dev Cell 2019;50:197-211.e5.

7. Basu R, Whitlock BM, Husson J, et al. Cells use mechanical force to potentiate target cell killing. Cell 2016; 165:100-110.

8. Bhat P, Leggatt G, Matthaei KI, and Frazer IH. The kinematics of cytotoxic lymphocytes influence their ability to kill target cells. PLoS One 2014;9:e95248. Erratum in: PLoS One 2014;9:e101381.

9. Birge RB, Boeltz S, Kumar S, et al. Phosphatidylserine is a global immunosuppressive signal in efferocytosis, infectious disease, and cancer. Cell Death Differ 2016;23:962-978.

10. Browne KA, Johnstone RW, Jans DA, and Trapani JA. Filamin (280-kDa actin-binding protein) is a caspase substrate and is also cleaved directly by the cytotoxic T lymphocyte protease granzyme B during apoptosis. J Biol Chem 2000;275:39262-39266.

11. Bufi N, Saitakis M, Dogniaux S, et al. Human primary immune cells exhibit distinct mechanical properties that are modified by inflammation. Biophys J 2015;108:2181-2190.

12. Cadart C, Zlotek-Zlotkiewicz E, Le Berre M, Piel M, and Matthews HK. Exploring the function of cell shape and size during mitosis. Dev Cell 2014;29:159-169.

13. Campestrini J, Silveira DB, and Pinto AR. HIV-1 Tatinduced bystander apoptosis in Jurkat cells involves unfolded protein responses. Cell Biochem Funct 2018;36: 377-386.

14. Caramalho I, Faroudi M, Padovan E, Müller S, and Valitutti S. Visualizing CTL/melanoma cell interactions: multiple hits must be delivered for tumour cell annihilation. J Cell Mol Med 2009;13(9B):3834-3846.

15. Carter GC, Bernstone L, Baskaran D, and James W. HIV-1 infects macrophages by exploiting an endocytic route dependent on dynamin, Rac1 and Pak1. Virology 2011;409: 234-250.
16. Chua BA, Ngo JA, Situ K, and Morizono K. Roles of phosphatidylserine exposed on the viral envelope and cell membrane in HIV-1 replication. Cell Commun Signal 2019; $17: 132$.

17. Comrie WA, and Burkhardt JK. Action and traction: cytoskeletal control of receptor triggering at the immunological synapse. Front Immunol 2016;7:68.

18. Crayne CB, Albeituni S, Nichols KE, and Cron RQ. The immunology of macrophage activation syndrome. Front Immunol 2019;10:119.

19. Davenport AJ, Cross RS, Watson KA, et al. Chimeric antigen receptor $\mathrm{T}$ cells form nonclassical and potent immune synapses driving rapid cytotoxicity. Proc Natl Acad Sci U S A 2018;115:E2068-E2076. Erratum in: Proc Natl Acad Sci U S A 2019;116:11075-11076.

20. Davis HW, Vallabhapurapu SD, Chu Z, et al. Enhanced phosphatidylserine-selective cancer therapy with irradiation and SapC-DOPS nanovesicles. Oncotarget 2019;10:856868.

21. Dehghani H, Puffer BA, Doms RW, and Hirsch VM. Unique pattern of convergent envelope evolution in simian immunodeficiency virus-infected rapid progressor macaques: association with CD4-independent usage of CCR5. J Virol 2003;77:6405-6418.

22. Desouza M, Gunning PW, and Stehn JR. The actin cytoskeleton as a sensor and mediator of apoptosis. Bioarchitecture 2012;2:75-87.

23. Elliott MR, and Ravichandran KS. The dynamics of apoptotic cell clearance. Dev Cell 2016;38:147-160.

24. Felce JH, and Dustin ML. Natural killers shed attachments to kill again. J Cell Biol 2018;217:2983-2985.

25. Feng Y, Brazin KN, Kobayashi E, et al. Mechanosensing drives acuity of $\alpha \beta$ T-cell recognition. Proc Natl Acad Sci U S A 2017;114:E8204-E8213.

26. Feng Y, Reinherz EL, and Lang MJ. $\alpha \beta$ T cell receptor mechanosensing forces out serial engagement. Trends Immunol 2018;39:596-609.

27. Franklin-Tong VE, and Gourlay CW. A role for actin in regulating apoptosis/programmed cell death: evidence spanning yeast, plants and animals. Biochem J 2008;413: 389-404.

28. Gadhamsetty S, Marée AF, de Boer RJ, and Beltman JB. Tissue dimensionality influences the functional response of cytotoxic T lymphocyte-mediated killing of targets. Front Immunol 2017;7:668.

29. Gadhamsetty S, Marée AFM, Beltman JB, and de Boer RJ. A sigmoid functional response emerges when cytotoxic $\mathrm{T}$ lymphocytes start killing fresh target cells. Biophys J 2017; 112:1221-1235.

30. Garg H, and Joshi A. Host and viral factors in HIVmediated bystander apoptosis. Viruses 2017;9:237.

31. Gidon-Jeangirard C, Hugel B, Holl V, et al. Annexin V delays apoptosis while exerting an external constraint preventing the release of CD4+ and PrPc+ membrane particles in a human T lymphocyte model. J Immunol 1999;162: 5712-5718.

32. Goping IS, Sawchuk T, Underhill DA, and Bleackley RC. Identification of $\{$ alpha $\}$-tubulin as a granzyme B substrate during CTL-mediated apoptosis. J Cell Sci 2006;119(Pt 5): 858-865.

33. Halle $\mathrm{S}$, Keyser KA, Stahl FR, et al. In vivo killing capacity of cytotoxic $\mathrm{T}$ cells is limited and involves dynamic interactions and T cell cooperativity. Immunity 2016;44:233245 . 
34. Hanke T, and Raulet DH. Cumulative inhibition of NK cells and $\mathrm{T}$ cells resulting from engagement of multiple inhibitory Ly49 receptors. J Immunol 2001;166:3002-3007.

35. Hivroz C, and Saitakis M. Biophysical aspects of T lymphocyte activation at the immune synapse. Front Immunol 2016;7:46.

36. Iyengar S, Hildreth JE, and Schwartz DH. Actin-dependent receptor colocalization required for human immunodeficiency virus entry into host cells. J Virol 1998;72:52515255.

37. Iyengar S, and Schwartz DH. Acquisition of CD4dependence by CD4-independent SIV passaged in human peripheral blood mononuclear cells. Retrovirology 2012;9: 61.

38. Iyengar S, and Schwartz DH. How do cell-free HIV virions avoid infecting dead-end host cells and cell fragments? AIDS Rev 2004;6:156-161.

39. Jenkins MR, Rudd-Schmidt JA, Lopez JA, et al. Failed CTL/NK cell killing and cytokine hypersecretion are directly linked through prolonged synapse time. J Exp Med 2015;212:307-317.

40. Jolly C, Kashefi K, Hollinshead M, and Sattentau QJ. HIV-1 cell to cell transfer across an Env-induced, actindependent synapse. J Exp Med 2004;199:283-293.

41. Jolly C, Mitar I, and Sattentau QJ. Requirement for an intact T-cell actin and tubulin cytoskeleton for efficient assembly and spread of human immunodeficiency virus type 1. J Virol 2007;81:5547-5560.

42. Kadolsky UD, and Yates AJ. How is the effectiveness of immune surveillance impacted by the spatial distribution of spreading infections? Philos Trans R Soc Lond B Biol Sci 2015;370:20140289.

43. Kim KS, Cho CH, Park EK, et al. AFM-detected apoptotic changes in morphology and biophysical property caused by paclitaxel in Ishikawa and HeLa cells. PLoS One 2012;7: e30066.

44. Kothakota S, Azuma T, Reinhard C, et al. Caspase-3generated fragment of gelsolin: effector of morphological change in apoptosis. Science 1997;278:294-298.

45. Kumari S, Mak M, Poh Y, et al. Irvine DJ Cytoskeletal tension actively sustains the migratory $\mathrm{T}$ cell synaptic contact. Cold Spring Harbor Laboratory bioRxiv 2018; DOI: http://dx.doi.org/10.1101/437236.

46. Kuwata $\mathrm{T}$, Byrum R, Whitted $\mathrm{S}$, et al. A rapid progressorspecific variant clone of Simian Immunodeficiency Virus replicates efficiently in vivo only in the absence of immune responses. J Virol 2007;81:8891-8904.

47. Kvansakul M. Viral infection and apoptosis. Viruses 2017; 9:356.

48. Le Floc'h A, Tanaka Y, Bantilan NS, et al. Annular PIP3 accumulation controls actin architecture and modulates cytotoxicity at the immunological synapse. J Exp Med 2013;210:2721-2737.

49. Lee YG, Chu H, Lu Y, et al. Regulation of CAR T cellmediated cytokine release syndrome-like toxicity using low molecular weight adapters. Nat Commun 2019;10:2681.

50. Lehmann MJ, Sherer NM, Marks CB, Pypaert M, and Mothes W. Actin- and myosin-driven movement of viruses along filopodia precedes their entry into cells. J Cell Biol 2005; 170:317-325.

51. Lichtner M, Marañón C, Vidalain PO, et al. HIV type 1infected dendritic cells induce apoptotic death in infected and uninfected primary CD4 T lymphocytes. AIDS Res Hum Retroviruses 2004;20:175-182.
52. Lin G, Lee B, Haggarty BS, Doms RW, and Hoxie JA. CD4-independent use of Rhesus CCR5 by human immunodeficiency virus Type 2 implicates an electrostatic interaction between the CCR5 $\mathrm{N}$ terminus and the gp120 C4 domain. J Virol 2001;75:10766-10778.

53. Liu Y, and Zhang T. Cell Softness Prevents Cytolytic T Cell Killing of Tumor-Repopulating Cells Available at SSRN: https://ssrn.com/abstract=3299430 or http://dx.doi .org/10.2139/ssrn.3299430 (Last accessed December 11, 2018).

54. Long EO, Kim HS, Liu D, Peterson ME, and Rajagopalan S. Controlling natural killer cell responses: integration of signals for activation and inhibition. Annu Rev Immunol 2013;31:227-258.

55. Mack M, Kleinschmidt A, Brühl H, et al. Transfer of the chemokine receptor CCR5 between cells by membranederived microparticles: a mechanism for cellular human immunodeficiency virus 1 infection. Nat Med 2000;6: 769-775.

56. Malinova D, Fritzsche M, Nowosad CR, et al. WASpdependent actin cytoskeleton stability at the dendritic cell immunological synapse is required for extensive, functional T cell contacts. J Leukoc Biol 2016;99:699-710.

57. Marchetti G, Gori A, Casabianca A, et al. Comparative analysis of T-cell turnover and homeostatic parameters in HIV-infected patients with discordant immune-virological responses to HAART. AIDS 2006;20:1727-1736.

58. Martin P, Pardo J, Schill N, et al. Granzyme B-induced and caspase 3-dependent cleavage of gelsolin by mouse cytotoxic T cells modifies cytoskeleton dynamics. J Biol Chem 2010;285:18918-18927.

59. Marzook NB, Latham SL, Lynn H, et al. Divergent roles of $\beta$ - and $\gamma$-actin isoforms during spread of vaccinia virus. Cytoskeleton (Hoboken) 2017;74:170-183.

60. Massanella M, Negredo E, Pérez-Alvarez N, et al. CD4 Tcell hyperactivation and susceptibility to cell death determine poor CD4 T-cell recovery during suppressive HAART. AIDS 2010;24:959-968.

61. Mohammadi P, Desfarges S, Bartha I, et al. 24 hours in the life of HIV-1 in a T cell line. PLoS Pathog 2013;9: e1003161. Erratum in: PLoS Pathog 2015;11:e1005006.

62. Morel PA, Faeder JR, Hawse WF, and Miskov-Zivanov N. Modeling the $\mathrm{T}$ cell immune response: a fascinating challenge. J Pharmacokinet Pharmacodyn 2014;41:401-413.

63. O'Connor RS, Hao X, Shen K, et al. Substrate rigidity regulates human $\mathrm{T}$ cell activation and proliferation. J Immunol 2012;189:1330-1339.

64. Pang B, Neijssen J, Qiao X, et al. Direct antigen presentation and gap junction mediated cross-presentation during apoptosis. J Immunol 2009;183:1083-1090.

65. Pang MF, Siedlik MJ, Han S, et al. Tissue stiffness and hypoxia modulate the integrin-linked kinase ILK to control breast cancer stem-like cells. Cancer Res 2016;76:52775287.

66. Raja J, Ludwig JM, Gettinger SN, Schalper KA, and Kim HS. Oncolytic virus immunotherapy: future prospects for oncology. J Immunother Cancer 2018;6:140.

67. Riedl S, Rinner B, Asslaber M, et al. In search of a novel target-phosphatidylserine exposed by non-apoptotic tumor cells and metastases of malignancies with poor treatment efficacy. Biochim Biophys Acta 2011;1808:26382645.

68. Roberts KL, and Baines JD. Actin in herpesvirus infection. Viruses 2011;3:336-346. 
69. Roozemond RC, and Bonavida B. Effect of altered membrane fluidity on NK cell-mediated cytotoxicity. I. Selective inhibition of the recognition or post recognition events in the cytolytic pathway of NK cells. J Immunol 1985;134: 2209-2214.

70. Roozemond RC, Das PK, and Halperin M. Effect of mycobacterial lipids on membrane fluidity and natural killer cell-mediated cytotoxicity. Ann Immunol (Paris) 1984; 135D:247-255.

71. Roozemond RC, Halperin M, and Das PK. Inhibition of natural killer cell-mediated cytotoxicity by lipids extracted from Mycobacterium bovis BCG. Clin Exp Immunol 1985; 62:482-490.

72. Roozemond RC, Mevissen M, Urli DC, and Bonavida B. Effect of altered membrane structure on NK cell-mediated cytotoxicity. III. Decreased susceptibility to natural killer cytotoxic factor (NKCF) and suppression of NKCF release by membrane rigidification. J Immunol 1987;139:17391746.

73. Rozmyslowicz T, Majka M, Kijowski J, et al. Platelet- and megakaryocyte-derived microparticles transfer CXCR4 receptor to CXCR4-null cells and make them susceptible to infection by X4-HIV. AIDS 2003;17:33-42.

74. Saha K, Zhang J, Gupta A, et al. Isolation of primary HIV1 that target CD8+ T lymphocytes using CD8 as a receptor. Nat Med 2001;7:65-72.

75. Santos LC, Blair DA, Kumari S, et al. Actin polymerizationdependent activation of Cas-L promotes immunological synapse stability. Immunol Cell Biol 2016;94:981-993.

76. Sibener LV, Fernandes RA, Kolawole EM, et al. Isolation of a structural mechanism for uncoupling $\mathrm{T}$ cell receptor signaling from peptide-MHC binding. Cell 2018;174:672687.e27.

77. Srpan K, Ambrose A, Karampatzakis A, et al. Shedding of CD16 disassembles the NK cell immune synapse and boosts serial engagement of target cells. J Cell Biol 2018; 217:3267-3283.

78. Stanton RJ, Prod'homme V, Purbhoo MA, et al. HCMV pUL135 remodels the actin cytoskeleton to impair immune recognition of infected cells. Cell Host Microbe 2014;16: 201-214.

79. Tamzalit F, Wang MS, Jin W, et al. Interfacial actin protrusions mechanically enhance killing bycytotoxic $\mathrm{T}$ cells. Sci Immunol 2019;4:eaav5445.

80. Taylor MP, Koyuncu OO, and Enquist LW. Subversion of the actin cytoskeleton during viral infection. Nat Rev Microbiol 2011;9:427-439.

81. Terry S, Savagner P, Ortiz-Cuaran S, et al. New insights into the role of EMT in tumor immune escape. Mol Oncol 2017;11:824-846.

82. Thammasri K, Rauhamäki S, Wang L, et al. Human parvovirus B19 induced apoptotic bodies contain altered self-antigens that are phagocytosed by antigen presenting cells. PLoS One 2013;8:e67179.

83. Théry M, and Bornens M. Get round and stiff for mitosis. HFSP J 2008;2:65-71.

84. Veselská R, Zitterbart K, Jelínková S, Neradil J, and Svoboda A. Specific cytoskeleton changes during apoptosis accompanying induced differentiation of HL-60 myeloid leukemia cells. Oncol Rep 2003;10:1049-1058.

85. Walsh D, and Naghavi MH. Exploitation of cytoskeletal networks during early viral infection. Trends Microbiol 2019;27:39-50.

86. Wang IH, Burckhardt CJ, Yakimovich A, and Greber UF. Imaging, tracking and computational analyses of virus entry and egress with the cytoskeleton. Viruses 2018;10:166.

87. Wang J, Zhang Y, Xu Q, et al. Menin mediates Tat-induced neuronal apoptosis in brain frontal cortex of SIV-infected macaques and in Tat-treated cells. Oncotarget 2017;8: 18082-18094.

88. Wang Y, Xu C, Jiang N, et al. Quantitative analysis of the cell-surface roughness and viscoelasticity for breast cancer cells discrimination using atomic force microscopy. Scanning 2016;38:558-563.

89. Wickman GR, Julian L, Mardilovich K, et al. Blebs produced by actin-myosin contraction during apoptosis release damageassociated molecular pattern proteins before secondary necrosis occurs. Cell Death Differ 2013;20:1293-1305.

90. Wodarz D. Cytotoxic T-lymphocyte memory, virus clearance and antigenic heterogeneity. Proc Biol Sci 2001;268: 429-436.

91. Wodarz D. Modeling $\mathrm{T}$ cell responses to antigenic challenge. J Pharmacokinet Pharmacodyn 2014;41:415-429.

92. Wu P, Zhang T, Liu B, et al. Mechano-regulation of peptide-MHC class I conformations determines TCR antigen recognition. Mol Cell 2019;73:1015-1027.e7.

93. Wurzer H, Hoffmann C, Al Absi A, and Thomas C. Actin cytoskeleton straddling the immunological synapse between cytotoxic lymphocytes and cancer cells. Cells 2019; $8: 463$.

94. Yu Y, Fay NC, Smoligovets AA, Wu HJ, and Groves JT. Myosin IIA modulates $\mathrm{T}$ cell receptor transport and CasL phosphorylation during early immunological synapse formation. PLoS One 2012;7:e30704.

95. Zaitseva E, Zaitsev E, Melikov K, et al. Fusion stage of HIV-1 entry depends on virus-induced cell surface exposure of phosphatidylserine. Cell Host Microbe 2017;22:99_ 110.e7.

Address correspondence to: Dr. David Schwartz

E-mail: dschwart@fastmail.fm or siyengar@fastmail.fm 